\title{
Riesgo de adicción a redes sociales, autoestima y autocontrol en estudiantes de secundaria
}

\author{
Risk of addiction to social networks, self-esteem and self-control in high school students
}

Katherine Challco Huaytalla ${ }^{1 *}$, Sheila Rodríguez Vega ${ }^{2}$, Jania Jaimes Soncco ${ }^{3}$

\begin{abstract}
RESUMEN
Objetivo: determinar la relación entre el riesgo de adicción a redes sociales, la autoestima y el autocontrol en estudiantes de 4to y 5to año del nivel secundario de una institución educativa pública de Lima Metropolitana. Material y métodos: La muestra estuvo conformada por 135 estudiantes con edades entre 14 y 16 años. Los instrumentos utilizados fueron el Cuestionario de Adicción a Redes Sociales (CARS), el Cuestionario de Autoestima de Coopersmith y el Cuestionario de Autocontrol Infantil y Adolescente (CACIA). Resultados: Se encontró que existe relación significativa inversa entre el riesgo de adicción a redes sociales, la autoestima y el autocontrol ( $\mathrm{r}=-.287 ; \mathrm{p}=.001 \mathrm{y} \mathrm{r}=-.288 ; \mathrm{p}=.001$; respectivamente), es decir a menor autoestima y autocontrol mayor será el riesgo de adicción a redes sociales. Conclusiones: a medida que los estudiantes muestren inseguridad, aislamiento, poco dominio propio sobre sus conductas, mayor será el riesgo de adicción a las redes sociales.
\end{abstract}

Palabras claves: Riesgo de adicción a redes sociales, autoestima, autocontrol, adolescentes.

\begin{abstract}
Objective: This research aimed to determine the relationship between the risk of addiction to social networks, self-esteem and self-control in students of 4th and 5th grade of high school of a public educational institution in Metropolitan Lima. Methods: The sample consisted by 135 teenagers between 14 and 16 years old. The instruments used were the questionnaire of addiction to social networks (CARS), the self-esteem questionnaire of Coopersmith and the questionnaire of infant self-control and adolescents (CACIA). Results: In the results were found that there is a significant trade-off between the risk of addiction to social networks, self-esteem and self-control ( $\mathrm{r}=-$. . 287; $\mathrm{p}=.001$ and $\mathrm{r}=-$. 288; $\mathrm{p}=.001$; respectively), That is to say, to lower self-esteem and self-control, greater will be the risk of addiction to social networks. Conclusions: The students show insecurity, isolation, low self-control over their conduct, the greater the risk of addiction to social networks.
\end{abstract}

Keywords: Risk of addiction to social networks, self-esteem, self-control, teens 


\section{INTRODUCCIÓN}

El internet posee ventajas extraordinarias para la vida en la actualidad, como la realización de actividades económicas, políticas y culturales, asimismo facilita la comunicación sincronizada a tiempo real por medio de las redes sociales (Castells, 2001). Sin embargo, el uso excesivo genera algunos trastornos psicológicos en los usuarios como la adicción a las redes sociales, el aislamiento, ansiedad por permanecer conectados y atentos a lo que suceda en las redes sociales, perdiendo la conciencia sobre el tiempo, olvidando realizar otras actividades y obligaciones, así mismo se desarrolla una sensación de temor y desconfianza frente a relaciones interpersonales presenciales, que pueden llegar a niveles patológicos (Garcés y Ramos, 2010).

Según Yen (2013), para la mayoría de las personas el concepto de adicción incluye usar drogas, por ello las definiciones sobre adicción se centran en el abuso de sustancias. Sin embargo, autores como Griffiths (citado por Yen, 2013) encuentran una serie de conductas potencialmente adictivas, incluyendo muchos comportamientos dentro de ellos el abuso de las redes sociales, definidas como adicciones tecnológicas, no químicas que involucran la interacción hombre-máquina, dejando de lado muchas veces las relaciones interpersonales.

En una encuesta de opinión pública, mencionan que la tasa de usuarios de internet en la población peruana se ha incrementado significativamente desde el año 2000 (9.7\%) hasta el 2011 (34.3\%). Señalando que dicha población da múltiples usos al internet, como herramienta de información, de interacción social y distracción, siendo usado mayormente por jóvenes y adolescentes, generando un impacto en sus estilos de vida (PUCP, 2010). En otra encuesta realizada por IPSOS (2014) se encontró que en el Perú existen $11^{\prime} 412,000$ millones de internautas siendo el 37\% de toda la población del Perú, el 83\% de internautas pertenece a alguna red social, el 99\% de la población urbana total entre 8 y 70 años tiene una cuenta en Facebook, siendo más del $50 \%$ jóvenes adultos entre 18 y 35 años.

En otro estudio Matalinares et al. (2013), encontraron que los adolescentes de la costa de Perú tienen mayor predisposición a ser adictos a internet debido a la facilidad de acceso, ya sea que tienen internet en casa o que pueden hallar con mayor facilidad cabinas de internet en comparación con adolescentes de la sierra y selva del Perú, siendo este un factor relevante para la conducta adictiva. Asimismo, encontró que el grupo de adolescente a con edades entre 15 a 16 años presenta mayor riesgo de adicción a internet. Esto provocaría en los adolescentes aislamiento, ansiedad, baja autoestima y pérdida de la capacidad de control (Echeburúa y Corral, 2010).

De acuerdo con Vilca y Gonzales (2013) la adicción de las redes sociales es una dependencia psicológica que a su vez muestra síntomas conductuales, afectivos y de pensamiento, siendo caracterizado principalmente por la falta de control en su uso. Tsitsika et al. (2013) agrega que es un patrón de conducta caracterizado por la falta de autocontrol. La persona adicta según Echeburúa y Coral (2010), se centra en los beneficios obtenidos inmediatamente, sin tener en cuenta las consecuencias negativas a largo plazo como pueden ser aislamiento y descuido del relacionamiento personal, de los deberes académicos e inclusive del descuido de la salud e higiene personal entre otros.

Existen diversos factores relacionados con el riesgo de adicción a las redes sociales, en este estudio se hace referencia a dos de ellas. En primer lugar está la autoestima que de acuerdo con Coopersmith (1967) es la evaluación que la persona realiza sobre sí misma, siendo consciente de sus límites, sus capacidades y su importancia como persona. Además es el juicio que se emite en cuanto a las actitudes de uno mismo, para cumplir las exigencias, conociendo las necesidades y carencias propias, aprendiendo a disfrutar el resultado del esfuerzo que se realiza en la vida diaria, siendo la experiencia parte esencial para llevar una vida significativa (Branden, 1995). En tal sentido Herrera, Pacheco, Palomar y Zavala (2010) encontraron que el uso constante de las redes sociales está relacionado con la depresión, la falta de habilidades sociales y la baja autoestima mostrando una percepción devaluada de su autoconcepto, su aspecto físico e intelectual, mostrándose indefensos y manteniendo una percepción deteriorada sobre sus relaciones interpersonales.

En segundo lugar, el autocontrol es otro factor que se relaciona con el riesgo de adicción a las redes sociales. Conforme a Capafons y Silva (2001) lo definen como un esfuerzo consciente del mismo sujeto por modificar sus acciones como también reacciones, influyendo en su ambiente y generando resultados ya sean positivos o negativos. Es también la regularización de emociones y realización de conductas pensando en las consecuencias (López; López y Freixinos, 2003). Asimismo es considerado como una característica de la personalidad que sirve como fuerza de voluntad, permitiéndole al sujeto controlar su propia conducta (Arana, 2014).

Por otro lado, en cuanto a la adolescencia Arab y Diaz (2014) consideran que es una etapa vulnerable 
a ciertas conductas de riesgo. Más aun Catalina, López de Ayala y García (2014) agregan que esto se debe a las características de este grupo de edad como son: la inmadurez, inestabilidad emocional e irresponsabilidad, así como, la búsqueda de la identidad, el sentido de pertenencia, sentimiento de omnipotencia, tendencia a ver a otros como los causantes de sus problemas, falta de experiencia en la vida, dificultad para reconocer y admitir una adicción y sensación de normalidad ante conductas de riesgo (Graner et al, 2007). Asimismo el internet se convirtió en un campo donde pueden explorar su propia identidad y sexualidad, experimentar nuevas emociones y relaciones sociales, sin embargo, lleva a la exposición de situaciones que pueden ser de riesgo para su seguridad física y mental, o crear conductas que pueden ser etiquetadas como problemáticas (Catalina, López de Ayala y García, 2014).

Por lo expuesto anteriormente, la presente investigación pretende determinar la relación entre el riesgo de adicción a las redes sociales, autoestima y el autocontrol en adolescentes de 4to y 5to año de secundaria de una institución pública de Lima.

\section{MATERIAL Y MÉTODOS}

La investigación es de enfoque cuantitativo, de diseño no experimental, de corte transversal y de alcance correlacional, (Hernández, Fernández y Baptista, 2010).

\section{Participantes}

Participaron 135 estudiantes con edades entre 14 y 16 años de ambos sexos, que se encontraban cursando el 4to y 5to año de secundaria de una institución educativa pública de Lima. Fueron seleccionados a través de un muestreo no probabilístico intencional, asimismo se solicitó el consentimiento informado a todos los participantes.

En la tabla 1 se puede observar que la muestra está compuesta en un 51,9\% por participantes de sexo masculino y un $48,1 \%$ de sexo femenino, asimismo el 57\% tiene 16 años y solo un 5,2\% tiene 14 años de edad, además se halló que el 54,1\% de participantes son estudiantes de 5to año de secundaria, Por otro lado el 83,7\% de participantes hacen uso de Facebook lo que indica que esta red social es la más usada por la muestra, con respecto a las horas que los educandos hacen uso de las redes sociales se reveló que el 60\% usa un tiempo menor de 3 horas.
Tabla 1

Datos sociodemográficos de la muestra

\begin{tabular}{llll}
\hline & & $\mathrm{n}$ & $\%$ \\
\hline Sexo & Masculino & 70 & 51.9 \\
& Femenino & 65 & 48.1 \\
Edad & 14 & 7 & 5.2 \\
& 15 & 51 & 37.8 \\
Grado & 16 & 77 & 57 \\
& 4 to & 62 & 45.9 \\
Red social & Fto & 73 & 54.1 \\
& Facebook & 113 & 83.7 \\
& WhatsApp & 10 & 7.4 \\
Tiempo de & YouTube & 4 & 3 \\
Uso & Otros & 8 & 5.9 \\
& Menos 3 horas & 81 & 60 \\
& De 4-6 horas & 31 & 23 \\
& De 7 a más horas & 23 & 17 \\
\hline
\end{tabular}

\section{Instrumentos}

Para el estudio se utilizó el cuestionario de Riesgo de Adicción a Redes Sociales (CARS) creado por Vilca y Gonzales (2013) en Lima-Perú, que mide la gravedad de síntomas y el nivel de riesgos de adicción a las redes sociales, conformada por siete dimensiones: pérdida de control, pérdida de interés por otras actividades, interferencia en el ámbito social, disminución del rendimiento académico, síndrome de abstinencia, modificación del ánimo y dependencia. Consta de 43 ítems con opciones de respuesta de tipo likert. Para este estudio la fiabilidad obtenida fue de un Alpha de Crombach de 0.92 para la escala general. La validez de constructo se realizó a través de la correlación Producto-Momento $r$ de Pearson encontrando significancia entre las dimensiones y el cuestionario general, lo que indica que es aceptable.

En cuanto al Cuestionario de Autoestima de Coopersmith creado en el año 1967 en California, se utilizó para el presente estudio la versión adaptada a Perú por Llerena en 1995 que mide el autoestima en 4 dimensiones: general de sí mismo, socialpares, hogar-padres y escuela, con un total de 58 ítems dentro de los cuales 8 pertenecen a la escala de mentira, con respuestas de tipo dicotómicos. En el estudio se obtuvo un Alpha de Crombach de 0,72 en la escala general indicando una buena fiabilidad, en sus dimensiones los valores obtenidos van desde 0,69 a 0,63 con excepción de las dimensiones social pares y escuela que obtuvieron puntajes bajos de 0,43 y 0,52 , por lo cual fueron retiradas. En cuanto a la validez se realizó la correlación Producto -Momento $r$ de Pearson entre las dimensiones de autoestima General de sí mismo y hogar - padres, mostrando una 
correlación altamente significativa, confirmando la validez de constructo.

Con relación al cuestionario de autocontrol infantil y adolescente (CACIA) el cuestionario original fue creado por Capafons y Silva en 1986 en Madrid, sin embargo para este estudio se usó la versión adaptada a Perú por Rodríguez en el 2009, con el fin de medir la capacidad de autocontrol adolescente en sus diferentes áreas. Consta de tres dimensiones: retroalimentación personal, retraso de la recompensa, autocontrol procesual y una escala de sinceridad, con un total de 71 ítems y opciones de respuesta de tipo dicotómico. La escala general del cuestionario en la muestra estudiada obtuvo un Alpha de Crombach de 0,71 indicando una buena fiabilidad, de igual forma sus tres dimensiones tiene una fiabilidad aceptable que van desde 0,64 a 0,75. En la validez se halló una correlación altamente significativa a través de la correlación Producto-Momento $r$ de Pearson indicando la validez del constructo.

Para el análisis se utilizó el software estadístico SPSS versión 20, asimismo se utilizó la prueba estadística de kolmogorov smirnov y el estadístico de correlación $r$ de Pearson.

\section{RESULTADOS}

Con el propósito de realizar los análisis de correlación y contrastar la hipótesis, se realizó la prueba de bondad de ajuste con el fin de ver si las variables presentan una distribución normal. En la tabla 2 se puede observar que las variables presentan una distribución normal debido a que el coeficiente (K$\mathrm{S})$ es significativo $(\mathrm{p}>0.05)$. Con relación al resultado obtenido, los análisis estadísticos correspondientes se realizarán a través de estadísticos paramétricos.

Tabla 2

Análisis de ajuste a la curva normal de las variables de estudio.

\begin{tabular}{lllll}
\hline Instrumento & Media & D.E & K-S & P \\
\hline $\begin{array}{l}\text { Riesgo de } \\
\text { redes sociales }\end{array}$ & 53,57 & 22,734 &, 910 &, 379 \\
Autoestima & 39,64 & 9,519 &, 887 &, 411 \\
Autocontrol & 34,76 & 5,855 &, 878 &, 423 \\
\hline
\end{tabular}

${ }^{*} p>0.05$

En cuanto a la correlación de Pearson en la tabla 3 se puede observar que existe una relación inversa altamente significativa entre el autocontrol, la autoestima y el riesgo de adicción a redes sociales ( $r=$ ,$- 288^{* *}$ y $r=-, 287 * *$ respectivamente). Esto indica que a menor autocontrol y autoestima, el riesgo de adicción a redes sociales es mayor. En cuanto a la correlación de las dimensiones de autocontrol y autoestima con el riesgo de adicción de redes sociales se observa que existe relación significativa con las dimensiones autocontrol procesual, si mismo general y hogar padres; a excepción de las dimensiones retroalimentación personal y retraso de la recompensa.

Tabla 3

Análisis de correlación entre el riesgo de adicción a redes sociales, el autocontrol y la autoestima.

\begin{tabular}{lll}
\hline & \multicolumn{2}{l}{$\begin{array}{l}\text { Riesgo de adicción a redes } \\
\text { sociales }\end{array}$} \\
\cline { 2 - 3 } & $r$ & $\mathrm{P}$ \\
\hline Autocontrol &,$- 288^{* *}$ & .001 \\
$\begin{array}{l}\text { Retroalimentación } \\
\text { personal }\end{array}$ &,- 165 & .056 \\
Autocontrol Procesual &,$- 242^{* *}$ & .005 \\
$\begin{array}{l}\text { Retraso de la } \\
\text { recompensa }\end{array}$ &,- 140 & .106 \\
Autoestima &,$- 287^{* *}$ & .001 \\
Si mismo general &,$- 229 * *$ & .008 \\
Hogar Padres &,$- 260 * *$ & .002 \\
\hline **. La correlación es significativa al nivel 0,01 & (bilateral).
\end{tabular}

\section{DISCUSIÓN}

En general se encontró que existe relación significativa inversa entre el riesgo de adicción a las redes sociales con la autoestima y el autocontrol $(r=-, 287$; p $=.001$ y $r=-, 288$; $\mathrm{p}=.001$ respectivamente) en los estudiantes de $4^{\circ}$ y $5^{\circ}$ año de secundaria, confirmando la hipótesis planteada. Es decir, a menor autoestima y autocontrol, mayor será el riesgo de adicción a las redes sociales y viceversa. En este contexto es importante señalar que los estudiantes con baja autoestima tienden a utilizar mayormente las redes sociales para interactuar con su entorno, ya que su inseguridad y cohibición social por temor al rechazo les impide hacerlo directamente, en este sentido son más propensos a la adicción de las redes sociales. Este resultado coincide con lo encontrado por Herrera et al. (2010), donde los jóvenes adictos a Facebook son más propensos a tener menos habilidades sociales y mostrar una autoestima baja. Por otro lado, los estudiantes con poco autocontrol, puede llegar a la adicción de las redes sociales por la falta de dominio en sus acciones, puesto que se centran en 
los beneficios inmediatos, como conversar con otros usuarios que poseen gustos y aficiones similares o comentarios positivos sobre acciones que realizan en su perfil virtual, sin embargo, si estos beneficios son continuos, habrán consecuencias negativas como el descuido de la salud e higiene, aislarse del mundo real y la adicción (Tsitsika et a., 2013; Echeburua y Coral, 2010).

Referente a la dimensión de si mismo general de la autoestima y el riesgo de adicción a las redes sociales se encontró que existe relación significativa inversa, es decir, a medida que los adolescentes tengan poca valoración y desconfianza de sí mismos, así como inseguridad en sus habilidades y atributos personales, presentaran mayor riesgo de adicción a las redes sociales. Entonces es razonable pensar que los adolescentes participantes de esta muestra que poseen una adecuada percepción de su persona, su imagen, sus habilidades y destrezas no necesitaran el comentario de terceros, ni compararse con otros a través de las redes sociales para afirmar la opinión que poseen de sí mismos debido a que tienen bien en claro quiénes son y cómo son, siendo esto un factor protector contra el riesgo de adicción a las redes sociales. Esta investigación difiere con el estudio de Peris, Maganto y Kortabarria (2013) donde mencionan que los adolescentes con alta autoestima relacionada al aspecto corporal y físico son quienes hacen mayor uso de las redes sociales indicando una relación positiva.

Respecto a la dimensión hogar-padres de la autoestima y la variable riesgo de adicción a las redes sociales en los estudiantes de $4^{\circ}$ y $5^{\circ}$ año de secundaria, el estadístico de $r$ Pearson muestra una correlación significativa inversa entre la dimensión y la variable, describiendo que a mayor interacción positiva del estudiante con los integrantes de la familia menor será el riesgo de adicción a redes sociales y viceversa. En esta dimensión se desarrollan las relaciones y cualidades con el entorno familiar, donde comparten aspiraciones y la percepción acerca de lo que está bien o mal, transmitiéndole al adolescente valores, actitudes y hábitos que aplicará diariamente (Piera, 2012). De este modo los estudiantes que tienen buenas relaciones con sus padres y hermanos no necesitan buscar seguridad y aceptación mediante la interacción con otras personas a través de las redes sociales, sin embargo, un entorno familiar conflictivo, padres rígidos, poco comprensivos o ausentes, convertirán el hogar en un ambiente hostil donde el estudiante tratará de alejarse buscando personas que puedan escucharlo o comprenderlo, recurriendo a uno de los accesos más cercanos e inmediatos como las redes sociales y la facilidad de interactuar con otras personas de diferentes lugares.
En cuanto a la dimensión retroalimentación personal del autocontrol y el riesgo de adicción a las redes sociales, se halló que no existe relación entre ambas. En este sentido los adolescentes que son poco cuidadosos de sus actos y consecuencias y que muestran poco interés por conocer las razones que determinan el por qué de sus actos, no estarían vinculados al riesgo de adicción a las redes sociales debido a que los resultados obtenidos en esta muestra son influenciados por un factor externo, siendo este el control ejercido por parte de los padres, quienes con mayor madurez ven con preocupación el futuro de sus hijos más aún si estos están en los últimos años de estudio. Esto es evidenciado a través de un estudio sobre el control y protección de los padres ante el uso de internet y redes sociales, donde menciona que son los padres quienes ejercen mayor control en cuanto al tiempo de conexión, siendo la madre quien muestra mayor interés y control por sus hijos (García y López, 2013).

Referente a la relación entre la dimensión retraso de la recompensa del autocontrol y la variable riesgo de adicción a las redes sociales, no existe relación entre la dimensión y la variable. Debido a que la dimensión se centra en el control de respuestas impulsivas, la priorización de actividades importantes, dejar de lado las actividades placenteras para cumplir con los deberes y mostrar una organización en sus responsabilidades y esto no está ligado al riesgo de adicción a redes sociales debido a factores externos como el control que ejercen los padres sobre los adolescentes, ya que la principal prioridad de cada padre es que sus hijos aprueben el año escolar, alejando los distractores que puedan obstaculizar el cumplimiento de sus prioridades, entre ellos el uso excesivo de las redes sociales. Esto es sustentado a través de teorías donde mencionan que los padres ejercen mayor control en esta etapa partiendo de las necesidades de sus hijos y centrándose en el cumplimiento de normas, responsabilidades (Fuentes, Motrico y Bersabe, 2003; Oliva, 2006; Montañés, Bartolomé, Montañés y Parra, 2008).

Finalmente se halló relación significativa inversa entre la dimensión autocontrol procesual y el riesgo de adicción a las redes sociales es decir a menor autocontrol procesual en los estudiantes de 4to y 5to de secundaria mayor será el riesgo de adicción a redes sociales, o viceversa. Es necesario comprender que el autocontrol procesual es la intranquilidad o disgusto por cuestionar el propio comportamiento así como la preocupación por actuar según las normas y reglas, de esta manera, los participantes de esta muestra con menor autocontrol procesual están caracterizados por el descuido y poco interés 
por el propio comportamiento y el cumplimiento de normas y reglas establecidas o la falta de estas, por lo tanto presentan mayor riesgo de adicción a las redes sociales. En este sentido, es lógico pensar que estos adolescentes debido a las características de su misma etapa tienden a mostrar poco interés por el cumplimiento de normas u horarios establecidos por los padres para hacer uso de las redes sociales, así como la falta de reglas y la facilidad de acceso a través del teléfono móvil sumado al poco tiempo dedicado al cuidado de los hijos debido a la carga laboral de los padres, son factores que contribuyen a un mayor riesgo de adicción a las redes sociales. Asimismo, Echeburúa (2012) sustenta que la rebeldía, la búsqueda de emociones fuertes y el deseo de transgredir las normas son características propias de la adolescencia. Por otro lado, los adolescentes están en mayor riesgo debido a que los padres tienen poco conocimiento sobre el uso de internet y pocas estrategias para una adecuada supervisión (Arab y Díaz, 2015).

\section{Declaración de financiamiento y de conflicto de intereses:}

El estudio fue financiado por los autores, quienes declaran no tener algún tipo de conflicto de interés en la investigación realizada.

\section{Correspondencia:}

Katherine Challco Huaytalla

Psicóloga del Colegio Adventista de Huaraz. Jr. Cajamarca N³50, Huaraz, Perú.

e-mail: katherine.challco@gmail.com

\section{REFERENCIAS BIBLIOGRÁFICAS}

Arab, E. y Díaz, A. (2015). Impacto de las redes sociales e internet en la adolescencia: Aspectos positivos y negativos. Revista Médica clínica Las Condes, 26(1), 7-13. Recuperado de http://www.elsevier.es/es-revistarevista-medica-clinica-las-condes-202-articuloimpacto-las-redes-sociales-e-90393596.

Branden, N. (1995). Los seis pilares de la autoestima. Barcelona: Paidos. Recuperado de http://www.gitanos. org/publicaciones/guiapromocionmujeres/pdf/04.pdf.

Capafons, A. y Silva, F. (2001) Cuestionario de autocontrol infantil $y$ adolescente (CACIA). Madrid: TEA ediciones.

Castells, M. (2001). La galaxia internet. España: Editorial Areté. Recuperado de http://baseddp.mec.gub.uy/ Documentos/Bibliodigi/La_galaxia_Internet.pdf

Catalina, B., López de Ayala, M. y García, A. (2014). Los riesgos de los adolescentes en internet: los menores como actores y victimas de os peligros de internet. Revista latina de comunicación social, Núm. 69, 462485. Recuperado de http://www.redalyc.org/articulo. oa?id=81931771008.

Coopersmith, S. (1967). The antecedents of selfesteem. San Francisco: Consulting Psychologists Press. Inc. Recuperado de https://books.google. com.pe/books?id=xvhGAAAAMAAJ\&dq=the $\% 20$ antecedents\%20of\%20self\%20esteem\&source=gbs_ book_other_versions.

Echeburúa, E. (2012). Factores de riesgo y factores de protección en la adicción a las nuevas tecnologías y redes sociales en jóvenes y adolescentes. Comunicaciones breves. 37 (4), 435-447 Recuperado de http://www.ehu. eus/echeburua/pdfs/RED\%20Adicci\%C3\%B3n\%20 nuevas\%20tecnolog\%C3\%ADas\%202012.pdf.
Echeburúa, E. y Paz de Corral. (2010). Adicción a las nuevas tecnologías y a las redes sociales en jóvenes: un nuevo reto. Adicciones, 22(2), 91-95. Recuperado de http://www.redalyc.org/articulo.oa?id=289122889001.

Encuesta de Opinión pública (Junio, 2010). Recuperado de: http://www.pucp.edu.pe/iop

Fuentes, M., Motrico, E. y Bersabe, R. (2003). Estrategias de socialización de los padres y conflictos entre padres e hijos en la adolescencia. Anuario de psicología, 34(3), 385-400. Recuperado de http://revistes.ub.edu/index. php/Anuario-psicologia/article/view/8739.

Garcés, J. y Ramos, M. (2010) Jóvenes consumidores y redes sociales en Castilla- La mancha. Castilla: Consejo de la Juventud de Castilla- La Mancha. Recuperado de:http:// www.portaljovenclm.com/documentos/noticias/3069/ JovenesConsumidoresyRedesSocialesenCLM.pdf no hay citación.

García, B., y López, M. (2013) Los padres ante el uso de internet y redes sociales. Trabajo presentado en el $\mathrm{V}$ congreso Internacional de Comunicación Social, España, Resumen recuperado de http://www. revistalatinacs.org/13SLCS/2013_actas/077_Catalina. pdf

Graner, P., Beranuv, M., et al. (2007) “¿Qué uso hacen los jóvenes y adolescentes de Internet y del móvil?” Comunicacióne Xuventude:Actas do Foro Internacional, 71-90. Recuperado el 7 de abril de 2008 en http:// dialnet.unirioja.es/servlet/articulo?codigo $=2648887$

Hernández, R., Fernández, C., \& Baptista, M. (2010). Metodología de la investigación. México: Mc Graw Hill. 
Herrera, M., Pacheco, M., Palomar, J. y Zavala, D. (2010). La adicción a Facebook relacionada con la baja autoestima, depresión y la falta de habilidades sociales. Psicología Iberoamericana, 18(1), 6-18. Recuperado de http://www.uia.mx/web/files/publicaciones/ripsic/ ripsic18-1.pdf.

Huayhuama, E. (2011). Clima social familiar, autoestima y estrategias de afrontamiento en adolescentes con y sin intento de suicidio. (Tesis para optar el título profesional de psicología). Universidad Nacional Mayor de San Marcos, Lima.

IPSOS Perú (2014) agencia de estudios de mercado e investigación especializada en Marketing, publicidad y opinión pública. http://www.ipsos-apoyo.com.pe/ tienda_estudios.

Lopez, C., Lopez, J. y Freixinos, M. (2003). Retardo de gratificación y autocontrol en jóvenes antisociales: características asociadas al género. Psicopatología clínica, legal y forense. 3(3), 5-21. Recuperado de http://www.masterforense.com/pdf/2003/2003art13. pdf.

Matalinares, M., Arenas, C., Díaz, G., Dioses, A., Yarlequé, L., Raymundo, O., Yaringaño, J. (2013). Adicción a internet y agresividad en estudiantes de secundaria del Perú. Revista IIPSI, Facultad de Psicología, UNMSM, 16(1), 75-93. Recuperado de http:// revistasinvestigacion.unmsm.edu.pe/index.php/psico/ article/viewFile/3920/3144.

Montañés, M., Bartolomé, R., Montañés, J. y Parra, M. (2008). Influencia del contexto familiar en las conductas adolescentes. Ensayos, Núm. 23, 391408. Recuperado de http://dialnet.unirioja.es/servlet/ articulo? codigo $=3003557$.

Oliva, A. (2006). Relaciones familiares y desarrollo adolescente. Anuario de psicología, 37(3), 209223. Recuperado de http://www.raco.cat/index.php/ AnuarioPsicologia/article/viewArticle/61838/.
Peris, M., Maganto, C. y Kortabarria, L. (2013). Autoestima corporal, publicaciones virtuales en las redes sociales y sexualidad en adolescentes. European Journal of Investigation in Health, Psychology and Education, 3(2), 171-180. Recuperado de file:///F:/Dialnet-Au toestimaCorporalPublicaciones VirtualesEnLasRed es-4519140.pdf.

Piera, N. (2012) Autoestima según género en estudiantes del quinto grado de la red educativa $N^{\circ} 4$, ventanilla. (Tesis para optar el grado académico de maestro en educación mención en psicopedagogía de la infancia). Recuperado de http://repositorio.usil.edu.pe/wpcontent/uploads/2014/07/2012_Piera_Autoestimaseg\%C3\%BAn-g\%C3\%A9nero-en-estudiantes-dequinto-grado-de-la-red-educativa-N\%C2\%B0-4Ventanilla.pdf.

Rodriguez, E. (2009). Autocontrol y hábitos de estudio en escolares de cuarto y quinto año de secundaria de Lima Metropolitana. (Tesis para optar el grado académico de magister con mención en psicología educativa) Universidad Nacional Mayor de San Marcos, Lima.

Tsitsika, A., Janikian, M., y Tzavela, E. (2013) Investigación sobre conductas adictivas a internet entre los adolescentes Europeos. Funded by the European Union. Recuperado de http://www.centrointernetsegura.es/ descargas/estudio_conductas_internet.pdf

Vilca, L y Gonzales, A. (2013). Propiedades psicométricas de la escala de Riesgo de Adicción a Redes Sociales (CARS) (tesis para optar el título profesional de psicólogo). Universidad Peruana Unión, Lima-Perú.

Yen, Y. (2013). Comunicación familiar y predisposición a la adicción a Facebook en adolescentes de instituciones educativas de Paita. Revista Nari Walac, Núm. 2. Recuperado de http://www.piuraheraldo.net/jspui/ handle/123456789/30.

Recibido: 26/02/2016

Aceptado: 15/04/2016 